\title{
Added Value of Individual Flexibility Profiles of Electric Vehicle Users For Ancillary Services
}

Andersen, Peter Bach; Sousa, Tiago; Thingvad, Andreas; Berthou, Lea Sass; Kulahci, Murat

Published in:

Proceedings of IEEE International Conference on Communications, Control, and Computing Technologies for Smart Grids

Link to article, DOI:

10.1109/SmartGridComm.2018.8587585

Publication date:

2018

Document Version

Peer reviewed version

Link back to DTU Orbit

Citation (APA):

Andersen, P. B., Sousa, T., Thingvad, A., Berthou, L. S., \& Kulahci, M. (2018). Added Value of Individual Flexibility Profiles of Electric Vehicle Users For Ancillary Services. In Proceedings of IEEE International Conference on Communications, Control, and Computing Technologies for Smart Grids IEEE. https://doi.org/10.1109/SmartGridComm.2018.8587585

\section{General rights}

Copyright and moral rights for the publications made accessible in the public portal are retained by the authors and/or other copyright owners and it is a condition of accessing publications that users recognise and abide by the legal requirements associated with these rights.

- Users may download and print one copy of any publication from the public portal for the purpose of private study or research.

- You may not further distribute the material or use it for any profit-making activity or commercial gain

- You may freely distribute the URL identifying the publication in the public portal 


\title{
Added Value of Individual Flexibility Profiles of Electric Vehicle Users For Ancillary Services
}

\author{
Peter Bach Andersen*, Tiago Sousa*, Andreas Thingvad*, Lea Sass Berthou*, Murat Kulahci ${ }^{\dagger}$ \\ *Department of Electrical Engineering, Technical University of Denmark, Lyngby \\ ${ }^{\dagger}$ Department of Applied Mathematics and Computer Science, Technical University of Denmark, Lyngby \\ ${ }^{\dagger}$ Department of Business Administration, Technology and Social Sciences, Lule University of Technology, Lule, Sweden
}

\begin{abstract}
Vehicle-Grid Integration (VGI) research may serve to limit the self-induced adverse effects of electric vehicles (EVs) in terms of additional grid loading, but also as to make the $\mathrm{EV}$ an active asset in supporting a stable, economic power system based on renewable energy. Any use of the vehicle for grid services requires an accurate understanding of the user's driving needs. This paper proposes the introduction of a user profile, describing the energy requirements for driving in terms of an energy deadline, target and minimum. To explore the use of such a profile, the paper analyses data from a Danish pilot project where the driving patterns of ten electric Nissan e-NV200 vans are investigated in terms of leave times and energy consumption. It is shown that the data can be fitted with a log-normal distribution that can be used to establish a per user profile which provides a certain statistical probability of fulfilling the driving needs while allowing an aggregator to optimize earnings. Initially, aggregators may apply similar driving assumptions across an entire fleet. Considering that the driving needs of individual EV owners are different, statistical representations of the individual behaviour may result in more flexibility, and thereby time, for providing grid services. The paper quantifies the value of such added flexibility based on the Danish market for frequency containment reserves.
\end{abstract}

\section{INTRODUCTION}

Electrification of the transportation sector will bring about both challenges and opportunities to the power system. The challenges will arise from the power system having to satisfy a new demand for power and energy in a safe and timely manner subject to a broad range of transportation needs. The opportunities are in using the electric vehicle (EV) as a flexible load and storage to not only limit the self-induced adverse effects in terms of additional grid loading, but also make the EV serve as an active asset in supporting a stable, economic power system based on renewable energy. These challenges and opportunities fall within Vehicle-Grid Integration (VGI) research and the cars that are designed with technical capabilities to support the power system can be called Grid Integrated Electric Vehicles (GIV). Using Electric Vehicle Supply Equipment (EVSE), enabling a bidirectional power exchange between EV and the grid referred to as Vehicle-To-Grid (V2G), may strengthen the EVs role in the power system.

Currently a portfolio of pilot projects is investigating the possibilities of VGI [1-4] with a growing emphasis on field testing with new series-produced, unmodified EVs supporting V2G. With an increased focus on field testing, and the general maturing of the technology, there is also a greater need to focus on the most important stakeholder in VGI research - the EV owner. It is prudent to understand if, and to what extend, the EV may be used as a resource to the grid without any adverse effects to the use for driving.

When an aggregator adds an EV to its portfolio, it may initially heavily prioritize the EV owner's driving needs as a safety measure. An initial guess on driving requirements may be based on other known customer profiles or direct inputs from the EV owner. The analysis of historic driving behavior may however allow the aggregator to define tailormade profiles over time to better explore user flexibility.

In the world's first commercial V2G pilot [5], at the utility company Frederiksberg Forsyning (FF) in Greater Copenhagen, the Parker project has access to more than a year worth of data from a company fleet of electric Nissan eNV200 vans, which are used for both daily field trips and for providing Frequency Containment Reserves (FCR) using V2G when parked. Based on this case study it is possible to define per user profiles for the ten e-NV200 drivers.

The main contributions of this study are as follows:

Firstly, the paper describes previous projects and studies focusing on EV driving data and owner involvement in charging management. The findings of these studies are then used when introducing a user profile with parameters determining the driving needs, and resulting flexibility, of an EV user.

Secondly, the FF pilot project is described along with the dataset used in this study.

Thirdly, an exploratory analysis based on FF fleet data is carried out for the selected parameters of the user profile. The assumed distributions for the parameters are described.

Finally, the paper explore the advantage of quantifying the per-user flexibility when offering an ancillary service product to the grid.

Ultimately the authors of this paper hope to contribute to VGI research by emphasizing the benefit, for both the user and the grid, in describing user behavior through data analysis.

\section{RELATED WORK}

This paper builds on previous studies on both EV service provision to the grid and EV usage pattern analysis, and the combination of the two when considering user-based constraints in optimization and dispatch strategies.

A considerable amount of work has already gone into describing the services an EV can provide to the power system. The investigation spans from services provided on a local 
level, aimed at the distribution system [6], to the services aimed at a larger regional level and the associated power and energy markets. One of the most profitable services currently investigated is that of Frequency Containment Reserve (FCR) [7]. Here an aggregated group of EVs provides frequency regulation through an aggregator where the EVs collectively respond to under- or over-frequencies by either charging or discharging. This use was first investigated by Kempton et al. $[8,9]$ in the US and has since been investigated for other regions including the Nordic synchronous zone [10-12]. This type of service is useful for understanding both the need and value of EV usage analysis. When providing frequency regulation an aggregator would be under a binding obligation to deliver a certain power capacity for a certain duration and need a detailed forecast on EV availability to make a suitable bid in the market. Since frequency regulation is a market based product, procured by the Transmission System Operator, it is possible to quantify earnings both in aggregate and per EV [7].

Concurrently, several studies have investigated the realworld usage patterns of EV drivers [13-16]. Such studies have aimed at uncovering both driving and plug-in patterns. For grid service provision specifically, such data is needed to define constraints in the optimization and dispatch methods defined for each service. Describing the exact requirements of the EV user will ensure that sufficient energy is available for driving while allowing aggregators to optimize their operation and earnings.

Initially, many service provision and optimization studies had to rely on simple assumptions on EV availability and user preferences or use constraints based on simulated data, e.g. using Monte Carlo simulations to get the data to reflect the stochastic nature of EV drivers [17]. While such assumptions and simulations may be sufficient to prove the feasibility and potential of certain services, field pilot studies as that of FF allow for a better understanding of EV usage.

Some research has gone into more detail with the specific parameters important for predicting the user behaviour. Kempton and Letendre [8] propose an intelligent chargedischarge control with three settings controlled by the user, which inspired the parameters used in this study. In [18] the authors describe end-user charging services which include a specific energy need and leave time per trip which has to either be found by manual input from the user or be predicted. By analyzing the actual behaviour profile, interview bias and manual inputs by the user can be avoided.

All these studies emphasize the importance of knowing the user behavior. To this end, the present study uses field data to analyze and assess the per user flexibility.

\section{USER PROFILE DEFINITION}

As soon as a 3rd party (e.g., an EV aggregator) influences the charging process of a vehicle, it becomes necessary to understand the implications it may have on the owner's driving needs. A user flexibility profile is essentially a set of parameters describing the driving needs of the user which have to be observed by an aggregator. Setting such parameters as accurately as possible helps guarantee driving requirements to be fulfilled, while allowing an aggregator to optimize its usage for grid services.

The aggregators use of the EV would require a number of 'known plugins', i.e., plugin sessions that recur in time and where both duration and energy needs are predictable in nature. Known plugins could for instance be 'night charging' or 'workplace charging'. Each EV owner would then have one or more known plugins which would each be described by the following parameters:

- Energy target that describes the quantity of energy needed between known plugins

- Energy deadline that describes the time where the Energy Target has to be met prior to the expected leave time of the vehicle.

- Minimum energy that describes a minimum energy threshold which has to be satisfied throughout the plug-in session.

These parameters are illustrated in Fig. 1. If an energy target is chosen, which accurately represents the needed energy, it will ensure sufficient range while reducing calender-based aging of the battery by not fully charging the battery [19]. The Minimum energy should be enough to cover an emergency trip to, for instance, the nearest hospital.

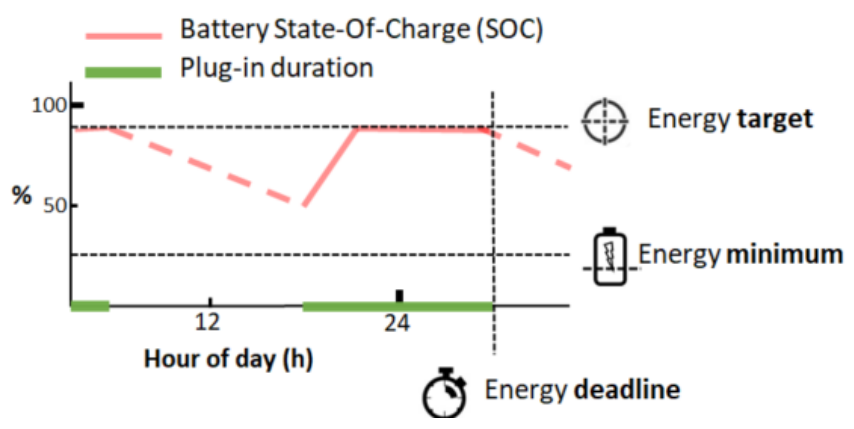

Fig. 1. User profile parameters

The three presented parameters are required in order to satisfy the EV owner's needs, but when selling a service, the aggregator also needs to know the full duration the EV will be plugged in and available. The plug-in time is therefore equally important as the other parameters for the aggregator, when it comes to optimizing the service provision.

If the EV owner knows that it is necessary to drive earlier or further than normally, it should be possible to override the service provision and charge at full power.

Ultimately a user profile may start with a conservative setting and then be gradually adjusted to the observed usage over time.

\section{DATA ANALYSIS}

This section starts by describing the FF dataset on which the analysis is based, this is followed by an exploratory analysis 


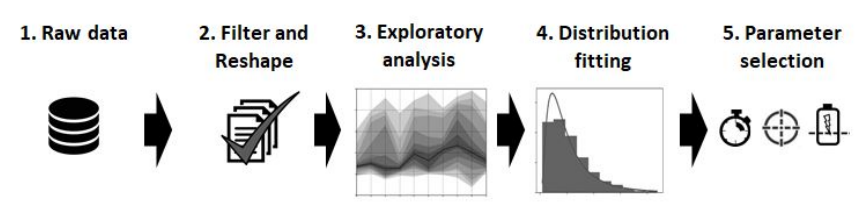

Fig. 2. Data analysis of FF data

describing the fleet's leave time and energy use. Finally, the data is fitted to a distribution which is used when selecting a specific energy deadline and target for a user profile. The steps of the data analysis are illustrated in Fig. 2.

\section{A. The dataset}

The data presented in this paper is collected from ten ENEL bi-directional DC $\pm 10 \mathrm{~kW}$ chargers owned by FF. Each charger is used by a specific driver as part of the utilities service fleet of ten electric Nissan e-NV200 vans (Fig. 3).

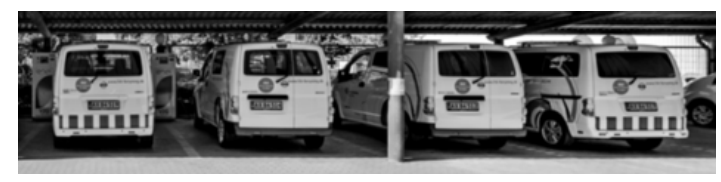

Fig. 3. e-NV200 vans at Frederiksberg Forsyning

In the following, each charger is numbered 1-10 with the prefix FF (FF01, FF02 etc.). Each charger record data for one specific vehicle and its user. Data from the EVSEs is logged every second and the total period considered is 480 days. The data includes a state variable describing whether a car is connected and the state-of-charge (SOC) of the battery, communicated in $\mathrm{kWh}$ via the CHAdeMO protocol.

These variables are used to define a number of trips for each EV. The leave and arrival times are based on changes in the EVSE state and the energy consumption based on changes in SOC. Since all data is recorded from the ten EVSEs the study only considers plug-ins at the company parking lot.

Outages and error-states of the EVSE have necessitated preprocessing the data to identify valid trips and remove faulty measurements. Out of the 480 days, the chargers have successfully logged around 450 days, except for charger FF08 that only has logged data for 290 days. The vehicles are only used during the workdays, which are close to 300 days. The number of logged days the EVs are driven are shown in Table I. It can be seen that the EVs are only driven half of the workdays on average, but with a large variance. The following

TABLE I

NUMBER OF DAYS WITH DRIVING OF THE DIFFERENT VEHICLES IN THE DATASET

\begin{tabular}{c|c|c|c|c|c|c|c|c|c} 
FF01 & FF02 & FF03 & FF04 & FF05 & FF06 & FF07 & FF08 & FF09 & FF10 \\
\hline 179 & 182 & 104 & 239 & 97 & 181 & 199 & 58 & 126 & 165
\end{tabular}

results are based on data consisting of the number of days seen in table I for each of the EVs.

\section{B. Exploratory analysis}

In Fig. 4 the leave time is plotted for each car with the mean and median values and percentiles. On average FF01 and FF03 leaves as the earliest and FF08 leaves the latest. The intervals around the median deviate in size, e.g. for car FF01, the interval is much smaller than for car FF09. By inspecting the $99.5 \%$ and $0.5 \%$ percentiles it is noted that there is a difference in the spread and therefore also in the uncertainty.

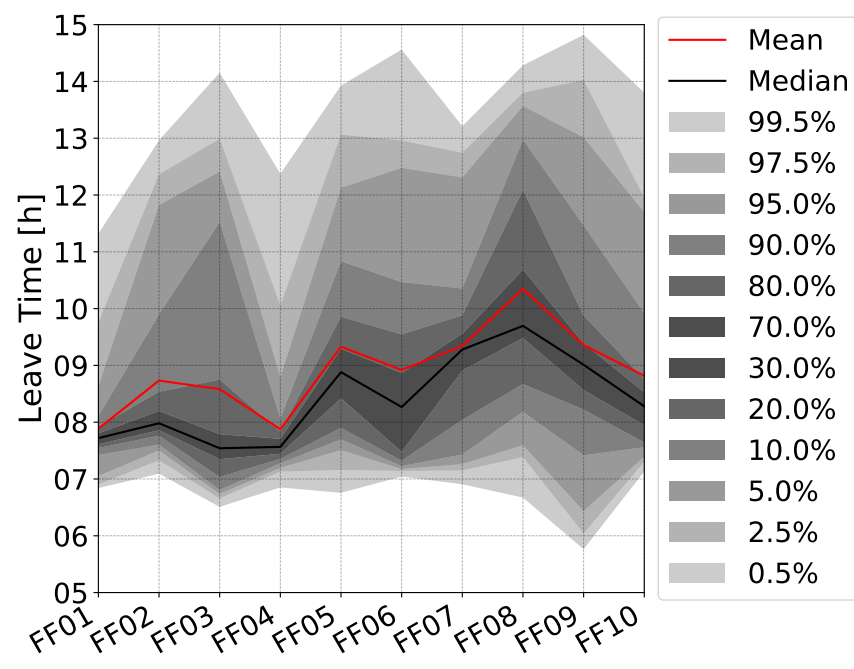

Fig. 4. The median and mean leave time for each car with percentiles.

In Fig. 5 the energy consumption of each EV is shown with the median and mean energy, and percentiles ranging from $99.5 \%$ to $0.5 \%$ are plotted for the individual cars. In general, the EVs have a similar median energy usage around $5 \mathrm{kWh}$, except for FF04 for which the usage is slightly higher and FF08 for which it is a little lower. FF07 has the largest 99.5\% percentile whereas the lowest percentiles do not differ much between the EVs. This daily energy usage is very low compared to the capacity of $24 \mathrm{kWh}$, which means that on average less than $1 / 4$ of the capacity is being used.

For both Fig. 4 and Fig. 5, the mean value is higher than the median and the percentiles also indicate that the data is positively skewed.

\section{Distributions}

The leave time and energy usage are fitted using a lognormal distribution. While other distributions could be considered (e.g. Weibull), log-normal is found to provide a sufficiently good fit for the purpose of this study. The histogram and fitted distribution for leave time are shown in Fig. 6. Here, only data from the first trip in a day is modelled due to the fact that the energy deadline should be aimed at the first trip. The leave time data is shifted by subtracting the earliest leave time for a better fit to the log-normal distribution. The actual leave time is then found by adding this time-shift again after fitting the distribution. For the whole fleet, the shifted time is 5 hours and 30 minutes. The next fitted variable is the energy usage, which is defined as the cumulative energy consumption 


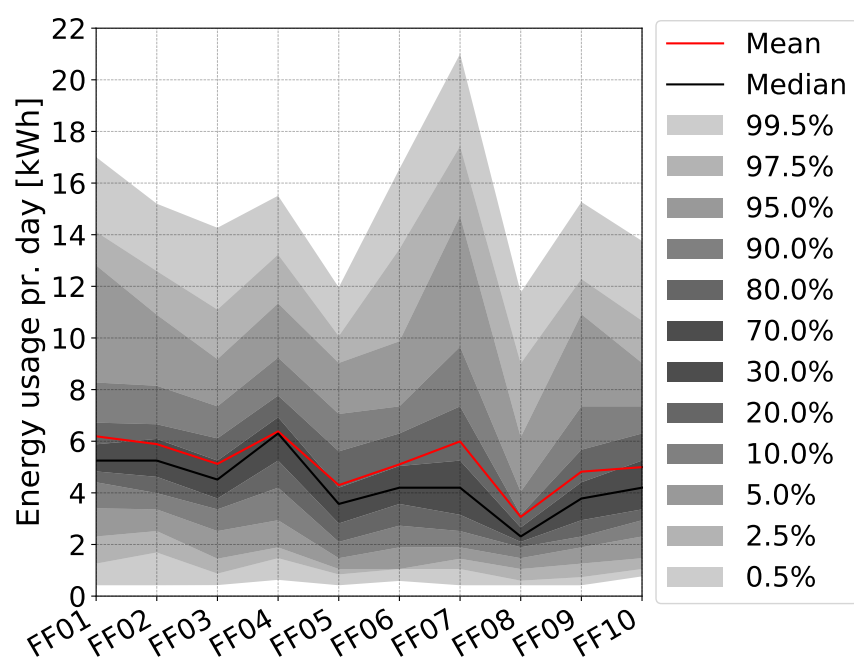

Fig. 5. Energy usage per car with percentiles

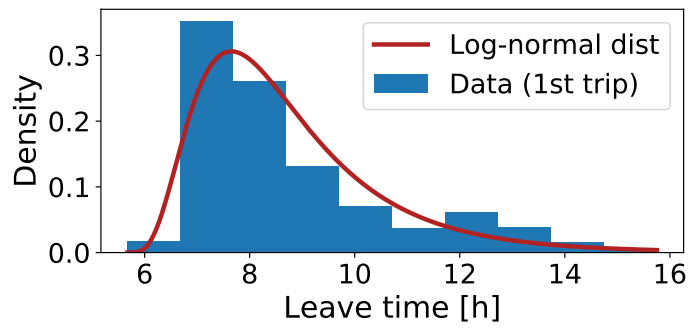

Fig. 6. Histogram of the leave time for the first trip in a day. The line shows the fitted log-normal function with $\mu=1.04$ and $\sigma=0.53$.

per day. For this fleet most of the EVs only have one main trip per day and, if any, the remaining trips are very short. The energy usage has a natural zero, which means that this data does not have to be shifted. A histogram and the fitted distribution for energy usage are shown in Fig. 7.

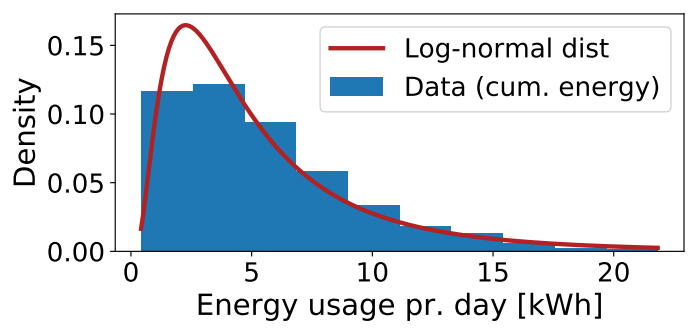

Fig. 7. Histogram of the cumulated energy usage per day. The line shows the fitted log-normal function with $\mu=1.44$ and $\sigma=0.79$.

\section{SElECTING PROFILES BASED ON PROBABILITY}

This section presents the Cumulative Distribution Function (CDF) for the FF fleet based on the log-normal distributions described previously. Afterwards we use the CDF for two vehicles to illustrate how specific energy targets and deadlines can be determined - and to show how the found values may differ and result in different degrees of flexibility. In Fig. 8 the CDF is shown for the energy need of the FF fleet. These distributions are fairly similar across the fleet, except EV FF08 which deviates by having a higher density for lower values and consequently could operate with a lower energy target. The energy need for the fleet is between $1 \mathrm{kWh}$ and $21 \mathrm{kWh}$ (99th percentile), where EV FF08 has a much smaller span between $1 \mathrm{kWh}$ and $13 \mathrm{kWh}$. The range of the energy need differs from what was observed in the raw data (Fig. 5) and is due to approximation errors in the log-normal fitting.

Fig. 9 shows the CDF for the leaving time of the fleet. The distributions vary more between the EVs, than the distributions of the energy need. The span of first trip leave times for the fleet is between 6:00 and 12:00. This spread is an indication that using individual energy deadlines may be especially beneficiary, compared to using a confidence interval of the whole group as we will see in section VI.

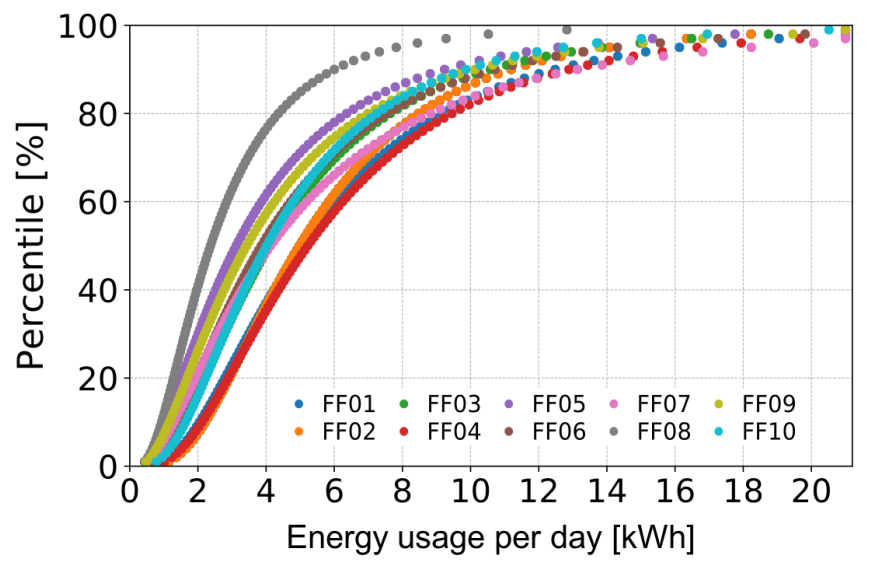

Fig. 8. CDF of the energy need

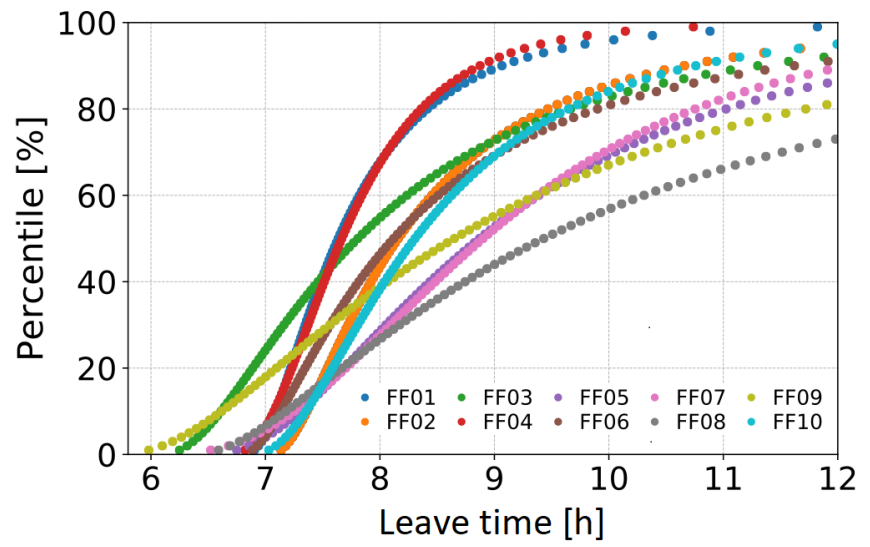

Fig. 9. CDF of the leave times

\section{A. Selecting and comparing flexibility profiles for two vehicles}

Out of the distributions presented in the last section we randomly choose two vehicles; FF04 and FF07. To arrive at 


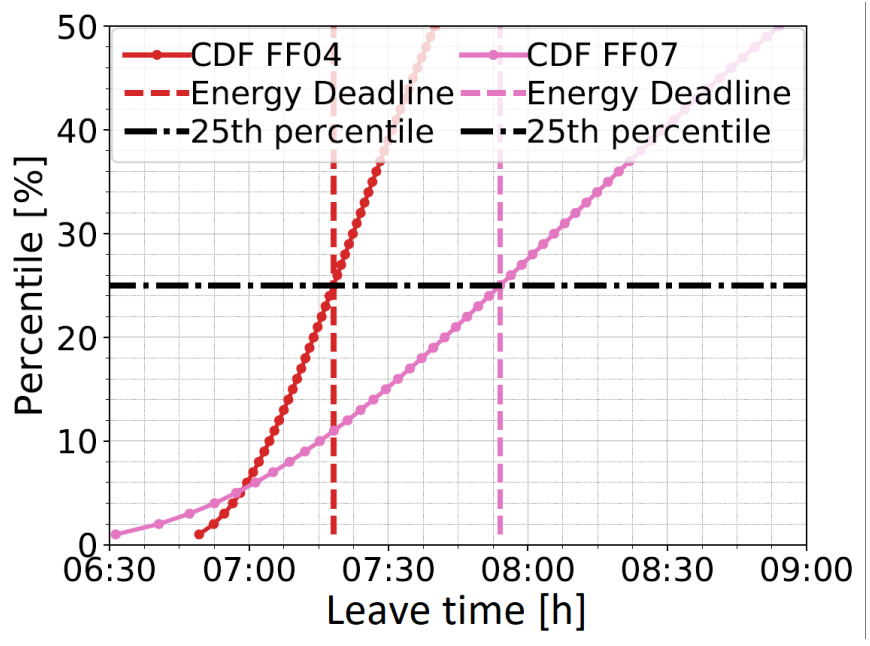

Fig. 10. CDF for the leave times of FF04 and FF07.

a specific energy deadline for each we choose an arbitrary percentile, in this case the 25 th percentile. Ultimately these percentiles reflect the trade off between using the vehicles longer in performing grid services and the risk/inconvenience faced by the owner. Selecting the 25th percentile for energy deadline represents a greater risk, as based on the distribution, a fourth of the leave times could happen prior to this time in the morning. The CDF for the leave times of both cars is depicted in Fig. 10.

It can be observed how choosing a low percentile results in FF07 having an earlier deadline than FF04, while a higher percentile gives a significantly later deadline to $\mathrm{FF} 07$, as the slope of the CDF is significantly lower. The trade-off between the inconvenience of the owner and the increased availability depends on the variance of the users leaving time. The deadlines found are 7:18 for FF04 and 7:54 for car FF07. The difference between the two energy deadlines means that FF07 will theoretically be available for 36 minutes longer to provide services than FF04. It can be seen that the two different distributions for the car, together with the percentile chosen, can have a great impact on the energy deadline found for each vehicle. The same approach as above can be used to identify the per vehicle energy targets. While the energy targets also will differ between vehicles it may not be as readily convertible to time available for grid services.

This simple comparison, based on leave times, shows that there are individual differences which may be worth exploiting for an aggregator providing frequency regulation services. In the next chapter we seek to quantify the value of knowing these individual profiles.

\section{ECONOMIC VALUE OF INDIVIDUAL PROFILING}

FCR is the most profitable service that can be delivered by EVs in combination with the $\pm 10 \mathrm{~kW}$ chargers at FF, as it is enumerated per power availability, not delivered energy. In the Nordic synchronous zone the service provider is obligated to deliver a proportional power response when the frequency deviates from $50 \mathrm{~Hz}$ with full power at deviations of \pm 100 $\mathrm{mHz}$. The service is symmetrical and requires availability of an equal amount of upwards and downwards regulation.

There are clear price patterns depending on the time of the day, with the highest prices during the night where fewest power plants are in operation [7]. The capacity payment is on average 35 EUR per MW available power per hour from 23:00 to 06:00 and, 25 EUR for the two hours before and after this period and 15 EUR the rest of the day.

The grid code requires guarantee of delivery, which means that failure to comply can result in a ban from the market.

There is uncertainty regarding the arrival time of the EV and the leaving time the next morning, which determines the service provision duration. Looking at the ten EVs as a group, they would in $99 \%$ of the days be plugged in at 18:00 and plugged out at 06:30, giving a 12:30 hour period. The 99th percentile of arriving and the 1st percentile of leaving, however looks different for the individual EVs than for the group, as seen in table II. By calculating the available period of each EV

TABLE II

ADDED CONNECTION TIME COMPARED WITH GROUP DISTRIBUTION WITH $99 \%$ PROBABILITY

\begin{tabular}{c|c|c|c|cc} 
& FF01 & FF02 & FF03 & FF04 & FF05 \\
\hline Plug in & $17: 45$ & $16: 45$ & $18: 00$ & $15: 50$ & $18: 15$ \\
Plug out & $06: 55$ & $07: 10$ & $06: 15$ & $06: 50$ & $06: 45$ \\
Added Time [min] & +10 & +85 & -15 & 150 & 0 \\
& FF06 & FF07 & FF08 & FF09 & FF10 \\
\hline Plug in & $17: 45$ & $16: 10$ & $17: 50$ & $18: 10$ & $17: 30$ \\
Plug out & $06: 55$ & $06: 30$ & $06: 35$ & $06: 00$ & $07: 00$ \\
Added Time [min] & +40 & +50 & -5 & -40 & +60
\end{tabular}

with a $99 \%$ confidence interval, the average period of service availability can be increased with 40 minutes, compared to calculating the same confidence interval of the whole group. If the average $\mathrm{EV}$ has an increased reserve provision time of 40 minutes per day with $\pm 10 \mathrm{~kW}$ corresponds to 60 EUR per year, assuming $25 \mathrm{EUR} / \mathrm{MW}$ per hour. If the EVs had individual owners, the most available EVs generate the highest revenues and should receive a higher compensation. The market is operated in hour blocks so the service can not be bid for 40 minutes but, aggregating a large pool of EVs, these partial hours can still be used.

Another uncertainty influencing the aggregation is that during FCR provision, the frequency can be too high or too low for extensive periods resulting in continuous charging or discharging. If ignored, it can lead to storage units such as batteries to become fully charged or depleted and thereby not able to deliver the service. Because of this, the aggregator can not bid the full power capacity for FCR as it is necessary to allocate part of the capacity to controlling the SOC [12]. The allocated power can maintain the SOC within certain limits while providing the reserve and if the energy target is low enough, the service can be delivered until the energy deadline without a period allocated for pure charging. A low energy target is preferable as it gives a larger acceptable range for the SOC to move depending on the frequency. 
Considering that the e-NV200 only has a $24 \mathrm{kWh}$ battery, it makes a large difference for the flexibility if the driver needs less than $21 \mathrm{kWh}$ or less than $12 \mathrm{kWh}$, as seen in Fig. 5. Up to one hour of additional reserve provision can be delivered if the EVs never charge to a level higher than the 99th percentile in Table III. One additional hour of reserve provision with $\pm 10 \mathrm{~kW}$ at the given time results in additional 90 EUR per year in revenue. The presented revenue is the pure

TABLE III

99TH PERCENTILE OF ENERGY REQUIREMENTS [KWH]

\begin{tabular}{c|c|c|c|c|c|c|c|c|c} 
FF01 & FF02 & FF03 & FF04 & FF05 & FF06 & FF07 & FF08 & FF09 & FF10 \\
\hline 17 & 15 & 14 & 15 & 12 & 16 & 21 & 12 & 15 & 14
\end{tabular}

capacity payment and not including costs of energy losses due to charger efficiency or wear of the battery.

\section{CONCLUSION}

Frederiksberg Forsyning is unique in that it is the first pilot project where EVs provide frequency regulation in a commercial setting. Consequently, it also represents a scenario where EV concurrently has to satisfy two critical applications, the drivers need of transportation and the bids placed in the FCR market by an aggregator.

To this end, this paper proposes defining a per user profile consisting of the three parameters, minimum energy, energy target and energy deadline. It is then shown how the latter two parameters may be found by considering a CDF based on data logged from the EV chargers.

This paper concludes by comparing the market availability, and related profit, using individual deadlines vs using the same energy deadline for the entire fleet. It is found that 40 minutes of additional reserve can be delivered per EV, using individual profiling with the same probability as when analyzing the whole group, resulting in 60 EUR per year increased revenue. It is also found that most of the EVs only need $60 \%$ SOC for the daily driving requirements, giving significantly more flexibility to the aggregator that does not need to reserve the high paying hours in the morning for normal charging, which can give an increase of revenue of up to 90 EUR per year.

This illustrates the advantage of moving from a conservative guess to using a tailor-made per vehicle profile with an energy target. The choice of percentile in the energy usage and leave time distributions, on which the energy deadline and target is based, reflect the risk-willingness of the user. Occasionally the EV user may be forced to either proactively overwrite the flexibility profile or accept having to wait for the EV to be sufficiently charged. Any occasional inconvenience must be related to the enumeration.

This study described the usage patterns of a specific commercial fleet of EVs. It is necessary to expand the study to other cases with other usage patterns.

Finally, there are seasonal changes in energy consumption for an EV which could be studied further, for this study the difference in energy consumption between summer and winter was not found to be significant.

\section{ACKNOWLEDGEMENTS}

The work in this paper has been supported by the research projects ACES (EUDP grant nr: EUDP17-I-12499) and Parker (ForskEL grant nr.

2016-1-12410). The data analysis was carried out by Lea Sass Berthou as part of her master thesis "Flexibility profiles for EV users" at DTU, 2018.

\section{REFERENCES}

[1] "Across continents electric vehicle services (aces)," http://acesbornholm.eu, 2017

[2] "The parker project," http://parker-project.com/, 2016.

[3] "Electric vehicle integration (invent)," 2017.

[4] "Gridmotion project: reducing electric vehicle usage cost thanks to smart charging process," 2017.

[5] M. N. B. Arias, J. Ostergaard, S. Hashemi, P. B. Andersen, C. Trholt, and R. Romero, "V2g enabled evs providing frequency containment reserves: Field results," in 2018 IEEE International Conference on Industrial Technology (ICIT 2018), 2018.

[6] K. Knezovic, S. Martinenas, P. B. Andersen, A. Zecchino, and M. Marinelli, "Enhancing the role of electric vehicles in the power grid: Field validation of multiple ancillary services," IEEE Transactions on Transportation Electrification, vol. 3, no. 1, pp. 201-209, March 2017.

[7] A. Thingvad, S. Martinenas, P. Andersen, M. Marinelli, B. Christensen, and O. Olesen, "Economic comparison of electric vehicles performing unidirectional and bidirectional frequency control in denmark with practical validation," 2016 Proceedings of the 51st International Universities Power Engineering Conference, 2016.

[8] W. Kempton and S. E. Letendre, "Electric vehicles as a new power source for electric utilities," Transportation Research Part D: Transport and Environment, vol. 2, no. 3, pp. 157 - 175, 1997.

[9] W. Kempton and J. Tomi, "Vehicle-to-grid power implementation: From stabilizing the grid to supporting large-scale renewable energy," Journal of Power Sources, vol. 144, no. 1, pp. 280 - 294, 2005

[10] S. Martinenas, M. Marinelli, P. B. Andersen, and C. Trholt, "Implementation and demonstration of grid frequency support by $\mathrm{v} 2 \mathrm{~g}$ enabled electric vehicle," in 2014 49th International Universities Power Engineering Conference (UPEC), Sept 2014, pp. 1-6.

[11] M. Marinelli, S. Martinenas, K. Knezovic, and P. B. Andersen, "Validating a centralized approach to primary frequency control with seriesproduced electric vehicles," Journal of Energy Storage, vol. 7, pp. 63 $73,2016$.

[12] A. Thingvad, C. Ziras, J. Hu, and M. Marinelli, "Assessing the energy content of system frequency and electric vehicle charging efficiency for ancillary service provision," in 2017 52nd International Universities Power Engineering Conference (UPEC), Aug 2017, pp. 1-6.

[13] Q. Wu, A. H. Nielsen, J. Ostergaard, S. T. Cha, F. Marra, Y. Chen, and C. Trholt, "Driving pattern analysis for electric vehicle (ev) grid integration study," in 2010 IEEE PES Innovative Smart Grid Technologies Conference Europe (ISGT Europe), Oct 2010, pp. 1-6.

[14] J. Quiros-Tortos, L. Ochoa, and B. Lees, "A statistical analysis of ev charging behavior in the uk," 102015.

[15] M. Neaimeh, G. Hill, P. Blythe, R. Wardle, J. Yi, and P. Taylor, "Integrating smart meter and electric vehicle charging data to predict distribution network impacts," in IEEE PES ISGT Europe 2013, Oct 2013, pp. 1-5.

[16] S. Speidel and T. Brunl, "Driving and charging patterns of electric vehicles for energy usage," Renewable and Sustainable Energy Reviews, vol. 40, pp. $97-110,2014$.

[17] A. S. B. Humayd and K. Bhattacharya, "Design of optimal incentives for smart charging considering utility-customer interactions and distribution systems impact," IEEE Transactions on Smart Grid, pp. 1-1, 2017.

[18] O. Sundstrm and C. Binding, "Charging service elements for an electric vehicle charging service provider," in 2011 IEEE Power and Energy Society General Meeting, July 2011, pp. 1-6.

[19] M. Ecker, N. Nieto, S. Kbitz, J. Schmalstieg, H. Blanke, A. Warnecke, and D. U. Sauer, "Calendar and cycle life study of li(nimnco)o2-based 18650 lithium-ion batteries," Journal of Power Sources, vol. 248, pp. $839-851,2014$ 